\title{
Bionic Surveillance System with a large field view
}

\author{
Huabo Sun ${ }^{\mathrm{a}}$, Jingru Han ${ }^{\mathrm{b}}$, Chun Wang ${ }^{\mathrm{c}}$, Yan Ma ${ }^{\mathrm{d}}$ \\ China Academy of Civil Aviation Science and Technology, Beijing, 100028, P.R.China \\ sunhb@castc.org.cn
}

Keywords: bionic surveillance, system composition, large field view.

\begin{abstract}
Usual security monitoring of civil airport generally uses fixed cameras to capture images and then to process them. There are some problems with performance including difficulties introduced in the information transmission, storage, and analysis of the process. Insect compound eyes offer a large field of view advantages for moving target capture and these have attracted the attention of many researchers in recent years. This paper contributes to this research by proposing a new surveillance system with large field of view applied in civil airport. We discuss the finished composition of the system, and introduce the proposed mathematical model of bionic compound eyes for data acquisition and image mosaic. Image matching and fusion algorithms for large view are also illustrated with different conditions. This algorithm effectively achieves safety surveillance of airport with a large field of view.
\end{abstract}

\section{Introduction}

Video surveillance of civil airport achieves development in recent years. Traditional fixed camera systems for surveillance dealt with the acquired images have several question, one significant is that the field of view is limited. During process acquired image, we must transform the three dimensional objects, which are acquired by high resolution sensors, to two dimensional images. Following this task one must then distinguish and locate the targets using signal-processing techniques. Finally we reverse the two-dimensional information to three-dimensions in order to redisplay the actual objects. The precision of detection and the amount of calculation required are positively correlated in such transformations. The higher the precision required the greater the amount of data involved. Consequently this delivers a less than real-time performance. As a result of these tradeoffs many researchers have turned their attention to a new image acquisition and processing mode called bionic compound eyes ${ }^{[1]}$. Tanida ${ }^{[2]}$ et al proposed a bionic compound eyes imaging system called TOMBO (Thin Observation Module by Bound Optics) which adapted micro lens and photo detectors for the acquisition and receiving assembly of light signals. This information is then processed to restructure the targets. Duparre ${ }^{[3]}$ et al proposed and manufactured the AACO (Artificial Apposition Compound Eye Objective $)^{[4]}$. Insect compound eyes have many advantages in moving target detection. They can detect and recognize targets in large areas using minimal information obtained through feature outline.

Insect compound eyes have many advantages in moving target detection. Insect compound eyes have small size, light weight, large viewing fields, and are very sensitive to moving targets. They can detect and recognize targets in large areas using minimal information obtained through feature outline. Insects can maintain high-resolution views of their targets while at the same time maintaining information about other targets in the same view. The mechanism of the compound eyes provides a solution to the tradeoffs between viewing field size, resolution, and real-time processing. These are very important problems that must be addressed when developing moving target detection systems. This paper follows on our previous work on moving object detection with bionic compound eyes applied in surveillance of airport ${ }^{[5]}$.

The remainder of this paper is organized as follows. In section 2 we explain our bionic surveillance system. Section 3 provides a report on some experimental analysis is discussed. Section 4 closes the paper with some conclusions and directions for future research. 


\section{Bionic surveillance system}

In this section we describe, in detail, the bionic surveillance system. The composition of the system is described in section 2.1. The biometric structure is described in section 2.2. Section 2.3 illustrates data acquisition model.

\subsection{Composition of the System}

Based on the mechanism of insect compound eyes for detecting moving targets, we have constructed a bionic compound eyes detection system. The compound eyes structure consists of the large field of view, a multi-angle mechanical structure, a hardware control system, a data acquisition device, and an object detection model. The "electrics" component illustrates the hardware used with electrical components for control, image sensing, processing, and data storage. The mechanical components of the system are the physical components making up the compound eyes structure.

\subsection{Biometric structure}

According to the physical structure of insect compound eyes the mechanical structure of the bionic compound eyes is constructed using high and low resolution cameras. Our compound eyes device, which is shown in Fig. 1, consists of five cameras. Four cameras surround one camera (central camera - high resolution) to form an equilateral hexagon. The fixed cameras devices are soft connected with the middle mechanical device. When detecting moving targets low resolution cameras detect the presence of moving objects while high resolution camera is responsible for target imaging. Using a servo system to control the angle of the cameras we can control the cameras to not only point to a specific position but also to monitor large areas in several directions.

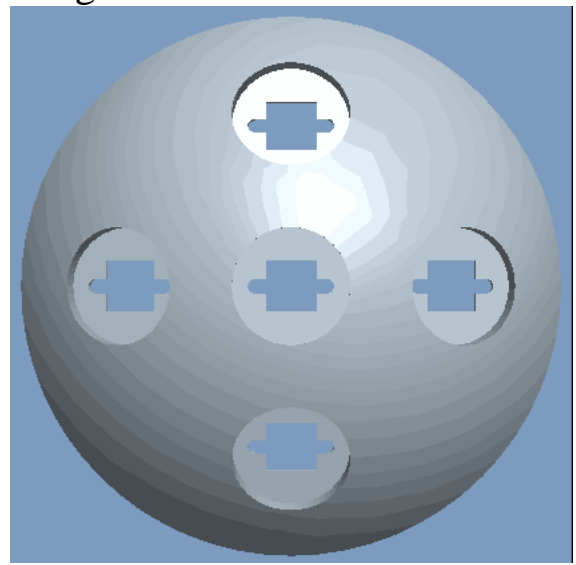

Figure 1. The physical structure of the five cameras on the compound eyes device.

As our system has a relatively large field of view the target will not quickly move out of visual range even if it is moving fast. Many authors agree with this approach such as Nanzhi et al ${ }^{[6]}$ who comment that this also greatly enhances the robustness of any moving target detection systems. Subsequently this increases the detection performance.

\subsection{Data acquisition model}

Compound eyes system has the advantage that several targets within a given range can be captured simultaneously using multiple sub-eyes. However these sub-eyes can introduce many redundant images. This potential disadvantage can be minimized by re-using these redundant images to assist in reducing the random error in the targets' information.

On the basis of previous algorithm we have developed an improvement to his algorithm by proposing a bi-level contour detection model with variable resolution bionic compound eyes, as is shown in Fig. 2. 


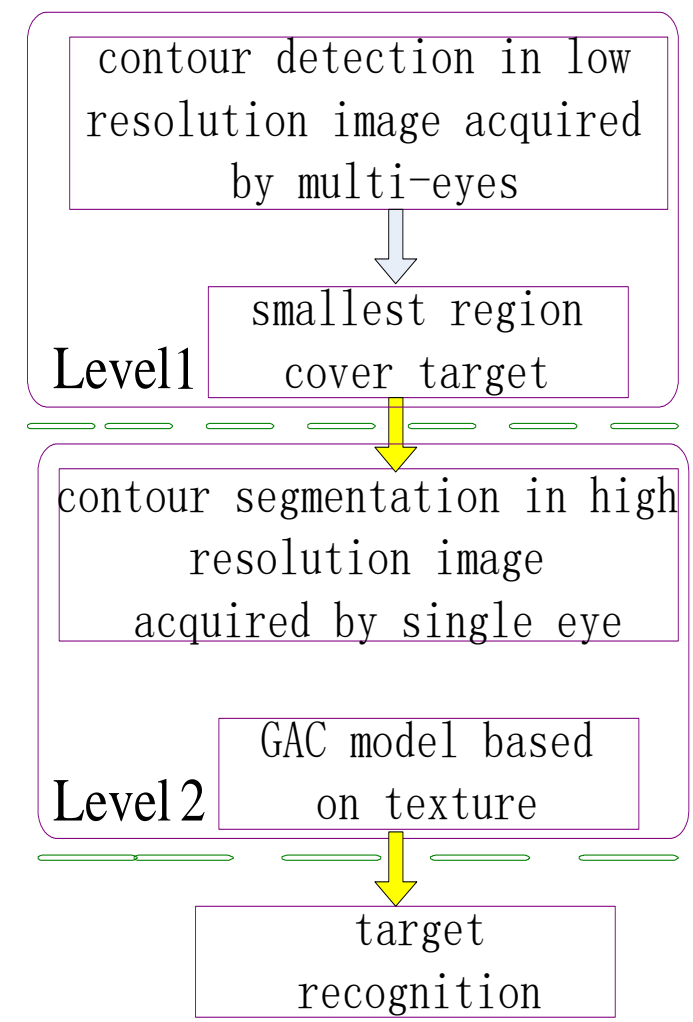

Figure 2. Flowchart diagram of the bi-level contour detection model used in our algorithm.

There are three important components of this model. Firstly there is contour detection which is applied to the lowest resolution images. When this is complete the texture gradient computation is performed by conducting contour segmentation on the high resolution image(s) acquired by the single eye. Finally a Geodesic Active Contour model for texture boundary detection is applied from which the moving target is recognized.

\section{Data process and result}

In this section we show an example of large view using bionic compound eyes. The example below shows the result processing the captured images using the approach SURF (Speeded-Up Robust Features) feature extraction, and then image registration and fusion are also described.

\subsection{Location feature points}

In this method, first of all, pre-classify the images according to the image geometric moment and the number of corner points, in order to achieve the automatic selection of image registration algorithm ${ }^{[7]}$ on the basis of the image features. SURF algorithm ${ }^{[8]}$ includes two parts: the feature detection and characterization. Feature characterization is described as

$$
v=\sum d_{x} \sum d_{y} \sum\left|d_{x}\right| \sum\left|d_{y}\right|
$$

Wherer $d_{x}$ is Haar wavelet response in the horizontal direction, $d_{y}$ is Haar wavelet response in the vertical direction. Feature detection is completed by the formula (2) and (3).

$$
\begin{aligned}
I_{\Sigma}(x) & =\sum_{i=0}^{i \leq x} \sum_{j=0}^{j \leq y} I(i, j) \\
H(x, \sigma) & =\left[\begin{array}{ll}
L_{x x}(x, \sigma) & L_{x y}(x, \sigma) \\
L_{y x}(x, \sigma) & L_{y y}(x, \sigma)
\end{array}\right]
\end{aligned}
$$

$I_{\Sigma}(x)$ stands for the sum of all the pixel gray values and $H_{(X, \sigma)}$ represents Hessian matrix. 


\subsection{Image Registration}
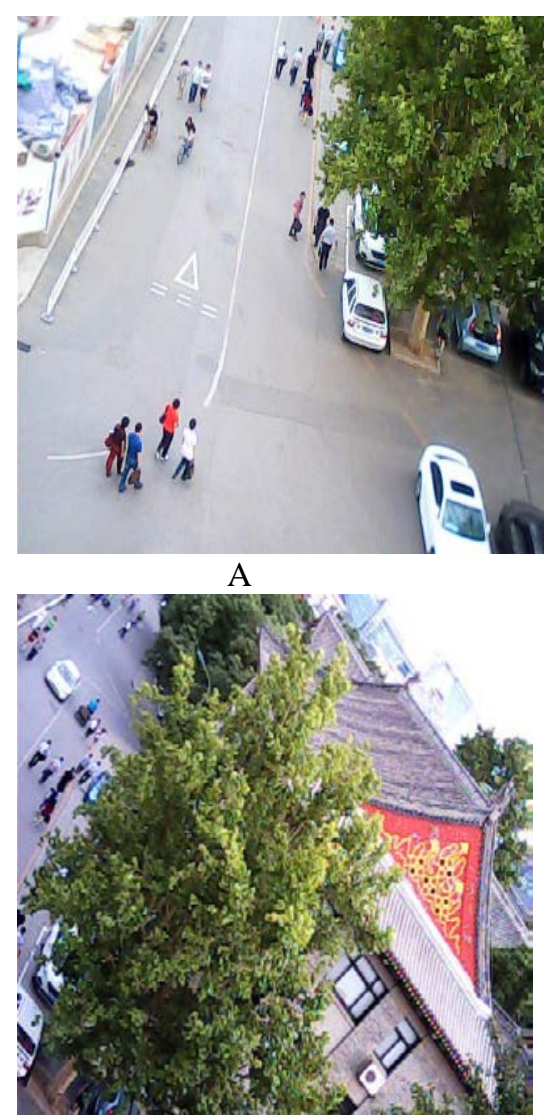

$\mathrm{D}$

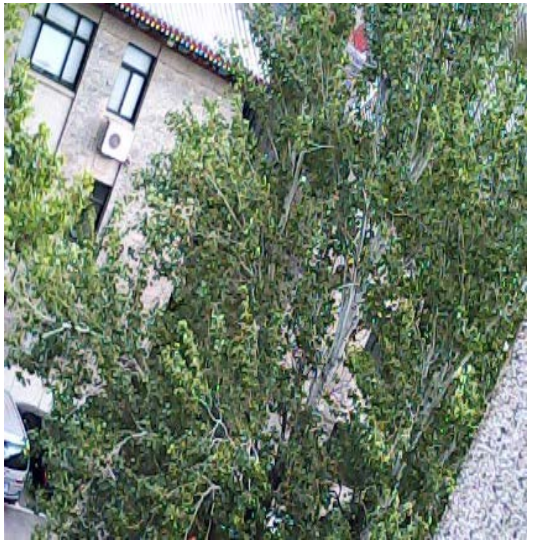

B

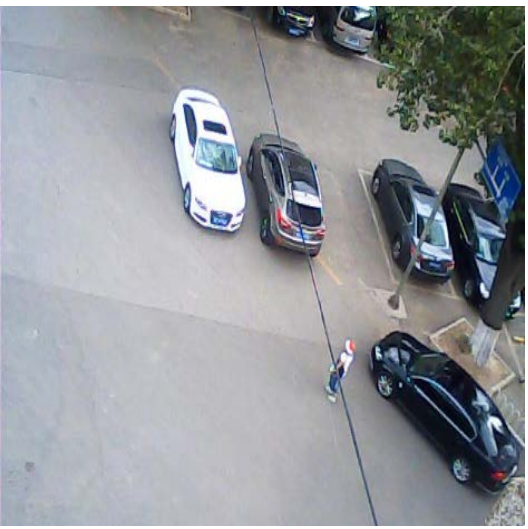

E

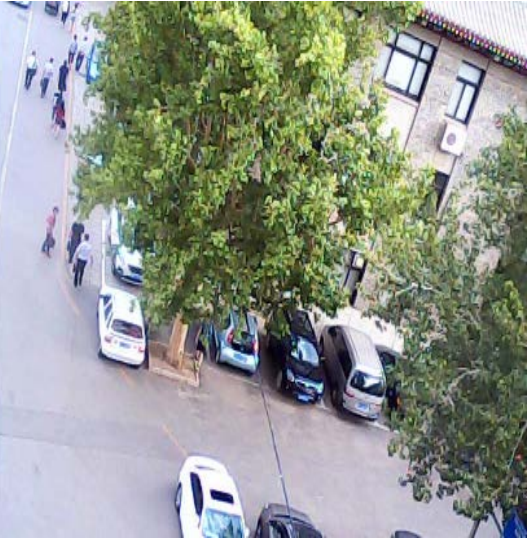

C
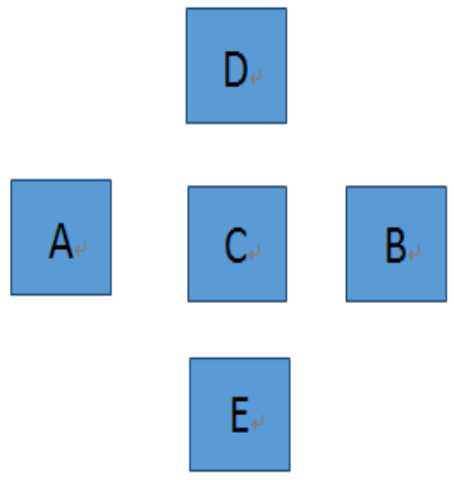

$\mathrm{F}$

Figure 3. The experimental data captured by Bionic surveillance system.

The images are processed by the RANSAC algorithm ${ }^{[9]}$, which includes several steps in the following:

1) 4 groups of matches are random selected to decided calculation Matrix $M$, and then calculate the distance of the remaining matches. If the distance is within the error range, the match is accepted point of M;

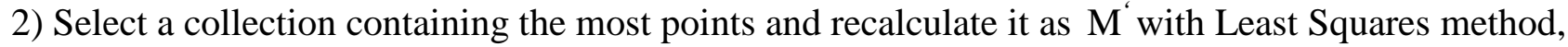
and remove a few outer points;

3) Repeat the above steps until get satisfied homographs.

The result of feature match is shown as Fig.4.

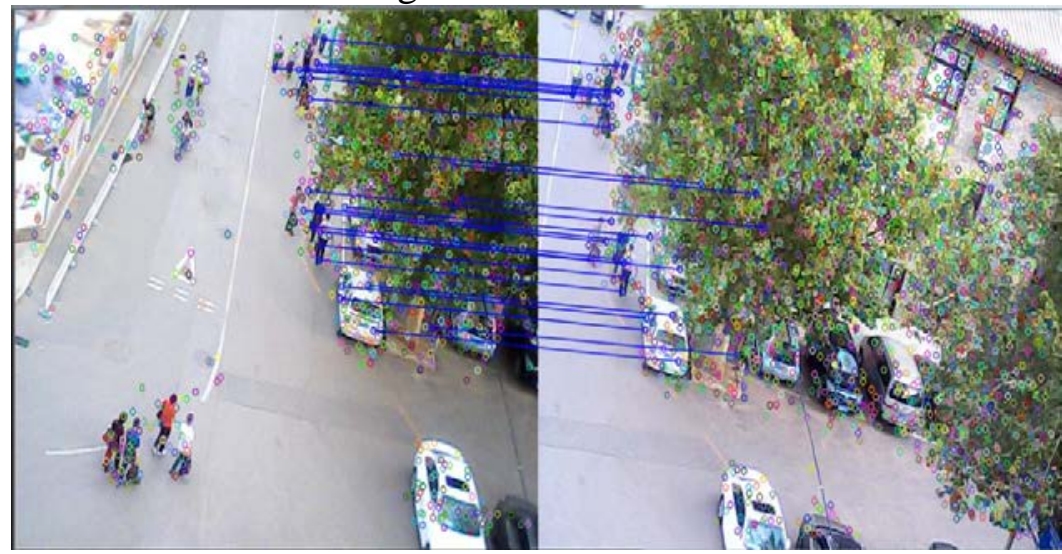

Figure 4. The result of feature match of image A\&C.

\subsection{Image Fusion}

Based on transformation matrix, we transformed images into the same image coordinates, and then using fade-out method for image fusion. Image fusion is described as 


$$
\begin{aligned}
& I(x, y)=\left\{\begin{array}{cc}
I_{1}(x, y) & (x, y) \in I_{1} \\
\partial I_{1}(x, y)+(1-\partial) I_{2}(x, y) & (x, y) \in I_{1}, I_{2} \\
I_{2}(x, y) & (x, y) \in I_{2}
\end{array}\right. \\
& \partial=\frac{W_{i}}{W}, \partial \in(0,1)
\end{aligned}
$$

Where $W_{i}$ is the horizontal distance between and the left edge of the two images overlap area, $W$ is the total width of the overlap region of the two images. It shows the contrast effect of direct mosaic and fade-out fusion in Fig.5 and Fig.6.

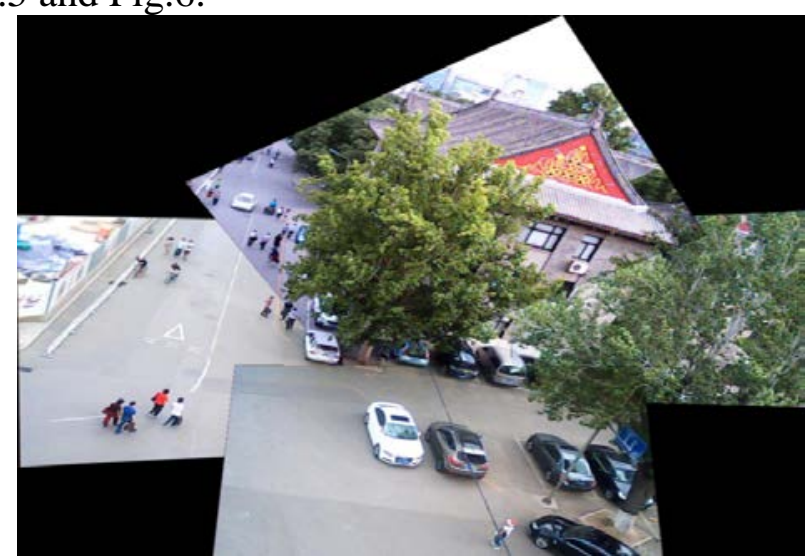

Figure 5. The result of direct mosaic.

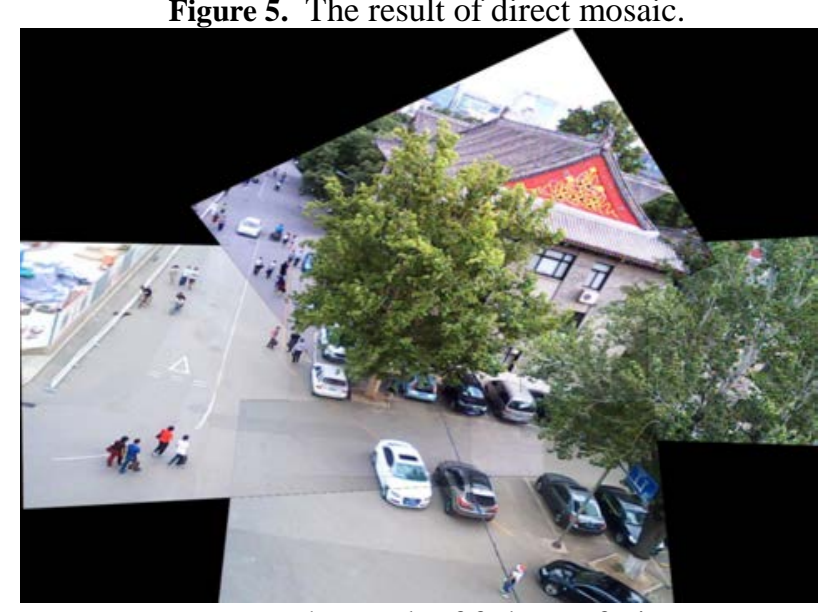

Figure 6. The result of fade-out fusion.

And then we using Inter-frame difference method to detect moving objects and get the initial result, shown as Fig.7.

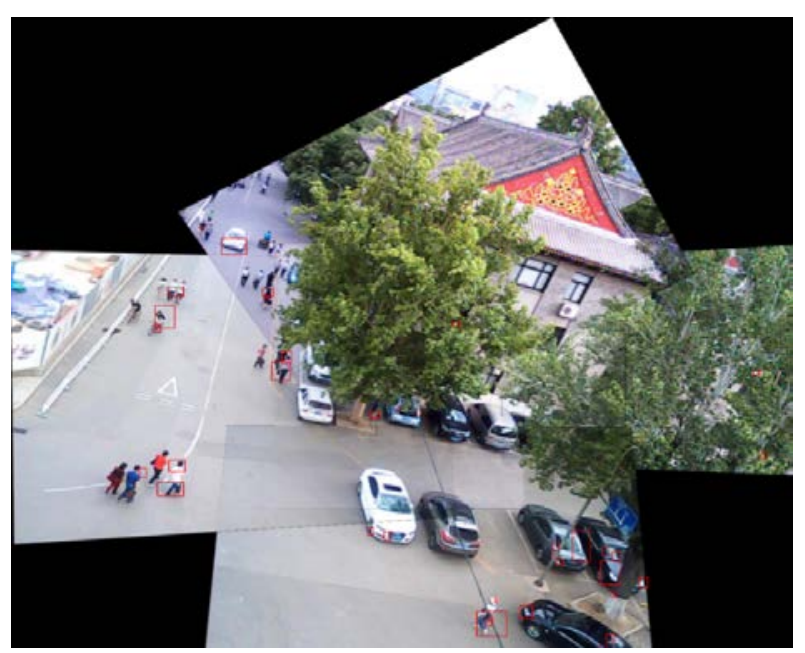

Figure 7. The result of moving target detection. 


\section{Conclusion}

The system we have described, in this paper, for surveillance using bionic compound eyes with a large field of view. Image matching and fusion algorithms for large view are also illustrated with different conditions. This system achieves a very satisfactory trade-off between the conflicting objects of: controlling the size of the field of view, the resolution of images captured, and the requirement to perform processing in real-time.

\section{Acknowledgment}

The work presented in this paper is supported by NSFC (National Natural Science Foundation of China) (ID: U1333124 \& U1533102). We thank the editor and reviewer for their contributions to improvements to this paper.

\section{References}

[1] Paragious N, Deirche R. Geodesic Active Contours and Level Sets for the Detection and Tracking of Moving Objects. IEEE Transactions on Pattern Analysis and Machine Intelligence, 22, 266-280(2000).

[2] Yamada K, Ishida S, Shougenji R, Tanida J. Development of three dimensional endoscope by Thin Observation by Bound Optics(TOMBO). Automation Congress, 199-205(2006).

[3] Duparré J, Wippermann F, Dannberg P, Bräuer A. Artificial compound eye zoom camera. Bioinspiration biomimetics, 3, 1806-1823(2008).

[4] Duparre J, Wippermann F, Darnnberg P, Schreiber P, Bräuer A, Völkel R, Scharf T. Micro-optical artificial compound eyes-from design to experimental verification of two different concepts. SPIE, 5962, 1-12(2005).

[5] Huabo Sun, Haimeng Zhao and Peter Mooney et al, A Novel System for Moving Object Detection Using Bionic Compound Eyes, Journal of Bionic Engineering, 8,313-322(2011).

[6] Nanzhi Jiang, Renbiao Wu, Jian Li. Super resolution feature extraction of moving targets. IEEE Transactions on Aerospace and Electronic Systems, 37, 781-793(2001).

[7] Bae K, Lichti D. A method for automated registration of unorganized point clouds[J]. ISPRS Journal of Photogrammetry and Remote Sensing, 63 (1): 36-54(2008).

[8] Ruan Qin, PengGang. Study on image registration and mosaic technology based on surf feature. Computer \& digital engineering, Vol.39, 141-144( 2011).

[9] XIE Kai, GUO Heng-ye, ZHANG Tian-wen. Image Mosaic technology summary [J]. Journal of Electronic, 32(7): 630-634(2004). 